\title{
Low Complexity Direction and Doppler Frequency Estimation for Bistatic MIMO Radar in Spatial Colored Noise
}

\author{
Lingyun Xu, ${ }^{1,2}$ Guangbin Zhang, ${ }^{1}$ Xiaofei Zhang, ${ }^{1}$ and Zongze $\mathrm{Xu}{ }^{1}$ \\ ${ }^{1}$ College of Science, Nanjing University of Aeronautics and Astronautics, Nanjing 210016, China \\ ${ }^{2}$ Nanjing Telecommunication Technology Institute, Nanjing 210007, China \\ Correspondence should be addressed to Guangbin Zhang; zhgb2007@nuaa.edu.cn
}

Received 5 November 2013; Revised 18 February 2014; Accepted 20 February 2014; Published 6 April 2014

Academic Editor: Shengqi Zhu

Copyright (c) 2014 Lingyun Xu et al. This is an open access article distributed under the Creative Commons Attribution License, which permits unrestricted use, distribution, and reproduction in any medium, provided the original work is properly cited.

\begin{abstract}
We investigate the algorithm of direction and Doppler frequency estimation for bistatic multiple-input multiple-output (MIMO) radar in spatial colored noise. A novel method of joint estimation of direction and Doppler frequency in spatial colored noise based on propagator method (PM) for bistatic MIMO radar is discussed. Utilizing the cross-correlation matrix which is formed by the adjacent outputs of match filter in the time domain, the special matrix is constructed to eliminate the influence of spatial colored noise. The proposed algorithm provides lower computational complexity and has very close parameters estimation compared to estimation of signal parameters via rotational invariance technique (ESPRIT) algorithm in high signal-to-noise ratio (SNR). It is applicable even if the transmitted waveforms are not orthogonal. The estimated parameters can be paired automatically and the Cramér-Rao Bound (CRB) is given in spatial colored noise. Simulation results confirm the effectiveness of the proposed method.
\end{abstract}

\section{Introduction}

Since multiple-input multiple-output (MIMO) radars use multiple antennas to simultaneously transmit diverse waveforms and utilize multiple antennas to receive the reflected signals, they have many potential advantages over conventional phased-array radars [1-4]. According to the array configuration, MIMO radar can be divided into two categories: the statistical MIMO radar and collocated MIMO radar [515]. The advantages of MIMO radar with collocated antennas have been studied extensively, which include improved direction performance and higher resolution [16], higher sensitivity or detection of moving targets, and increased degrees of freedom for transmit beamforming [17]. MIMO radar with widely separated antennas can capture the spatial diversity of the target's radar cross-section (RCS) [18]. This spatial diversity provides radar system with the ability to improve target parameter estimation [19-22], high resolution target localization, and tracking performance $[23,24]$.

Target direction estimation is a basic function of a radar system. Many advanced direction estimation algorithms for MIMO radar have been extensively discussed in the current literature which include ESPRIT algorithm, Capon algorithm, parallel factor (PARAFAC) algorithm, multiple signal classification (MUSIC) algorithm, and PM algorithm [2533]. In [25, 29], ESPRIT algorithm exploited the invariance property of both the transmit array and the receive array for direction estimation in MIMO radar systems. Reference [30] derived a reduced-dimension multiple signal classification (MUSIC) algorithm for direction of departure (DOD) and direction of arrival (DOA) estimation. The algorithm, which only requires one-dimensional search, can avoid the high computational cost of the two-dimensional MUSIC algorithm. However, the mentioned algorithm above did not consider the Doppler frequency estimation, and the noises were assumed to be the Gaussian white noise. In [31], the ESPRIT method was used for DOD-DOA and Doppler frequency estimation which necessitates eigen decomposition of the sample covariance matrix. Huge computation will be involved where the large array size is required in applications. Yunhe [32] proposed the DOA matrix algorithm to estimate the DOD-DOA and Doppler frequency, but it cannot eliminate the influence of spatial colored noise. In this paper, we propose a low-complexity angle and Doppler frequency 
estimation algorithm which can reduce computational cost. It has very close parameters estimation performance compared to ESRPIT and DOA matrix algorithm in high SNR. And the proposed algorithm pairs the parameters automatically and eliminates the influence of the spatial colored noise. Simulation results illustrate performance of the proposed algorithm.

The remainder of this paper is structured as follows. Section 2 develops the data model for a bistatic MIMO radar system, and Section 3 proposes the proposed algorithm for angle and Doppler frequency estimation in MIMO radar. In Section 4 , simulation results are presented to verify improvement for the proposed algorithm, while the conclusions are shown in Section 5.

Notation. $(\cdot)^{*},(\cdot)^{T},(\cdot)^{H},(\cdot)^{-1},(\cdot)^{\dagger}$, and vec $[\cdot]$ denote complex conjugation, transpose, conjugate-transpose, inverse, Moore-Penrose inverse (pseudoinverse), and vectorization operator, respectively. \|\|$_{\mathrm{F}}$ presents Frobenius norm; $\mathbf{I}_{K}$ is a $K \times K$ identity matrix; $\otimes$ represents Kronecker product; $\min (\cdot)$ is to get minimum elements of an array; angle $(\cdot)$ denotes the phase of a complex; $\operatorname{row}(\cdot)$ denotes the operator that stacks the rows of a matrix in a column vector. $D_{n}(\cdot)$ is to take the $n$th row of the matrix to construct a diagonal matrix. $E[\cdot]$ is expectation operator.

\section{Data Model}

We consider a narrowband bistatic MIMO radar system with $M$-element transmit antennas and $N$-element receive antennas, both of which are half-wavelength spaced uniform linear arrays. The transmit antennas transmit $M$ orthogonal coded signals. $\mathbf{s}_{m}=\left[s_{m}(1), s_{m}(2), \ldots, s_{m}(P)\right]^{T} \in \mathbf{C}^{P \times 1}, m=$ $1,2, \ldots, M$ denotes the sampled baseband coded signal of the $m$ th transmit antenna with one repetition interval with $P$ being the length of the transmitted code sequence, so the transmit signals can be expressed as $\mathbf{S}=\left[\mathbf{s}_{1}, \mathbf{s}_{2}, \ldots, \mathbf{s}_{M}\right]^{T}$. We also assume that there are a number of $K$ far-field independent targets; $\mathbf{a}_{r}\left(\theta_{k}\right)$ and $\mathbf{a}_{t}\left(\phi_{k}\right)$ are the receive steering vector and transmit steering vector for $\theta_{k}(\mathrm{DOA})$ and $\phi_{k}$ (DOD) of the $k$ th target, so the arrival signal of the $k$ th target is $\mathbf{a}_{t}^{T}\left(\phi_{k}\right) \mathbf{S}$. The received array through reflections of the target can be expressed as

$$
\mathbf{Y}(t)=\sum_{k=1}^{K} \beta_{k} e^{j 2 \pi f_{d k}(t-1) / f_{s}} \mathbf{a}_{r}\left(\theta_{k}\right) \mathbf{a}_{t}^{T}\left(\phi_{k}\right) \mathbf{S}+\mathbf{W}(t),
$$

where $\beta_{k}$ and $f_{d k}$ denote the radar cross-section (RCS) fading coefficient and Doppler frequency of the $k$ th target. $f_{s}$ is the pulse repeat frequency. Due to the steering vector of ULA, we have $\mathbf{a}_{r}\left(\theta_{k}\right)=\left[1, e^{-j \pi \sin \theta_{k}}, \ldots, e^{-j \pi(N-1) \sin \theta_{k}}\right]^{T}, \mathbf{a}_{t}\left(\phi_{k}\right)=$ $\left[1, e^{-j \pi \sin \phi_{k}}, \ldots, e^{-j \pi(M-1) \sin \phi_{k}}\right]^{T}$.

$\mathbf{w}(t) \in \mathbf{C}^{N \times P}$ denotes a Gaussian noise of zeros mean with unknown covariance matrix $\mathbf{Q}_{w}$. Matching the received data with the signal $(1 / \sqrt{P}) \mathbf{S}^{H}$, we obtain

$$
\mathbf{x}(t)=\mathbf{A} \boldsymbol{\eta}(t)+\mathbf{n}(t)
$$

where $\mathbf{A}=\left[\overline{\mathbf{a}}_{1}, \overline{\mathbf{a}}_{2}, \ldots, \overline{\mathbf{a}}_{K}\right]$ is an $M N \times K$ matrix composed of the $K$ steering vectors, and $\overline{\mathbf{a}}_{k}=\mathbf{a}_{r}\left(\theta_{k}\right) \otimes \overline{\mathbf{a}}_{t}\left(\phi_{k}\right)$ is the Kronecker product of the receive and the transmit steering vectors for the $k$ th target. $\overline{\mathbf{a}}_{t}\left(\phi_{k}\right)=\mathbf{R}_{s}^{T} \mathbf{a}_{t}\left(\phi_{k}\right)$, owing to $\mathbf{R}_{s}=$ $E\left[\mathbf{S S}^{H} / P\right]=\mathbf{I}_{M}$; then $\overline{\mathbf{a}}_{t}\left(\phi_{k}\right)=\mathbf{a}_{t}\left(\phi_{k}\right) \cdot \boldsymbol{\eta}(t)=\left[\eta_{1}(t), \eta_{2}(t)\right.$, $\left.\ldots, \eta_{K}(t)\right]^{T}, \eta_{k}(t)=\sqrt{P} \beta_{k} e^{j 2 \pi f_{d k}(t-1) / f_{s}}, k=1,2, \ldots, K \cdot \mathbf{n}(t)=$ $\operatorname{vec}\left[\mathbf{W}(t) \mathbf{S}^{H} / \sqrt{P}\right]$.

The covariance matrix of $\mathbf{n}(t)$ is as follows:

$$
\begin{aligned}
& E\left[\mathbf{n}(i) \mathbf{n}^{H}(j)\right] \\
& =\frac{E\left\{\operatorname{vec}\left[\mathbf{W}(i) \mathbf{S}^{H}\right] \operatorname{vec}^{H}\left[\mathbf{W}(j) \mathbf{S}^{H}\right]\right\}}{P} \\
& =\frac{E\left\{\left[\mathbf{S}^{*} \otimes \mathbf{I}_{N}\right]\left[\operatorname{vec}(\mathbf{W}(i)) \operatorname{vec}^{H}(\mathbf{W}(j))\right]\left[\mathbf{S}^{T} \otimes \mathbf{I}_{N}\right]\right\}}{P} \\
& = \begin{cases}\mathbf{I}_{M} \otimes \mathbf{Q}_{w}, & i=j \\
\mathbf{0}, & i \neq j .\end{cases}
\end{aligned}
$$

\section{Direction and Doppler Frequency Estimation Algorithm for MIMO Radar}

3.1. The Proposed Algorithm Description. We assume that $\mathbf{a}_{t}\left(\phi_{k}\right)$ and $\mathbf{a}_{r}\left(\theta_{k}\right)$ are constant for $L$ samples and define $\mathbf{X}$ as $\mathbf{X}=[\mathbf{x}(1), \mathbf{x}(2), \ldots, \mathbf{x}(L)]$, and we assume that the number of snapshots is $L$. Let

$$
\begin{gathered}
\mathbf{X}_{1}=[\mathbf{x}(1), \mathbf{x}(2), \ldots, \mathbf{x}(L-1)]=\mathbf{A} \boldsymbol{\eta}_{1}+\mathbf{N}_{1}, \\
\mathbf{X}_{2}=[\mathbf{x}(2), \mathbf{x}(3), \ldots, \mathbf{x}(L)]=\mathbf{A} \boldsymbol{\eta}_{2}+\mathbf{N}_{2},
\end{gathered}
$$

where $\boldsymbol{\eta}_{1}=[\boldsymbol{\eta}(1), \boldsymbol{\eta}(2), \ldots, \boldsymbol{\eta}(L-1)], \boldsymbol{\eta}_{2}=[\boldsymbol{\eta}(2), \boldsymbol{\eta}(3)$ $, \ldots, \boldsymbol{\eta}(\mathrm{L})], \mathbf{N}_{1}=[\mathbf{n}(1), \mathbf{n}(2), \ldots, \mathbf{n}(L-1)]$, and $\mathbf{N}_{2}=$ $[\mathbf{n}(2), \mathbf{n}(3), \ldots, \mathbf{n}(\mathrm{L})]$. Equation (3) shows that the crosscovariance matrix of noises is $\mathbf{0}$. This characteristic will be utilized in this paper to improve the estimate performance.

Note that $E\left[\mathbf{N}_{2} \mathbf{N}_{1}^{H}\right]=0, \boldsymbol{\eta}_{2}=\boldsymbol{\Phi} \boldsymbol{\eta}_{1}, \boldsymbol{\Phi}=\operatorname{diag}\left[e^{j 2 \pi f_{d 1} / f_{s}}\right.$, $\left.e^{j 2 \pi f_{d 2} / f_{s}}, \ldots, e^{j 2 \pi f_{d K} / f_{s}}\right]$. This indicates that the rotation factor $\Phi$ is generated by adjacent outputs of match filters.

The covariance matrix of $\mathbf{X}_{1}$ and $\mathbf{X}_{2}$ can be written as follows:

$$
\mathbf{R}_{x}=E\left[\mathbf{X}_{2} \mathbf{X}_{1}^{H}\right]=\mathbf{A} \Phi \mathbf{R}_{\eta} \mathbf{A}^{H}+E\left[\mathbf{N}_{2} \mathbf{N}_{1}^{H}\right]=\mathbf{A} \Phi \mathbf{R}_{\eta} \mathbf{A}^{H},
$$

where $\mathbf{R}_{\eta}=E\left[\boldsymbol{\eta}_{1} \boldsymbol{\eta}_{1}^{H}\right]$. For the independent targets, $\mathbf{R}_{\eta}$ should be a diagonal matrix; then we have the relationship $\mathbf{R}_{\eta} \boldsymbol{\Phi}^{H}=$ $\boldsymbol{\Phi}^{H} \mathbf{R}_{\eta}$. Owing to $E\left[\mathbf{N}_{2} \mathbf{N}_{1}^{H}\right]=0, \mathbf{R}_{x}$ can eliminate the influence of spatial colored noise. A new matrix $\mathbf{R}$ is constructed by utilizing (5):

$$
\mathbf{R}=\left[\begin{array}{c}
\mathbf{R}_{x}^{H} \\
\mathbf{R}_{x}
\end{array}\right]=\left[\begin{array}{c}
\mathbf{A} \Phi^{H} \\
\mathbf{A} \boldsymbol{\Phi}
\end{array}\right] \mathbf{R}_{\eta} \mathbf{A}^{H}=\underbrace{\left[\begin{array}{c}
\mathbf{A} \Phi^{-1} \\
\mathbf{A} \boldsymbol{\Phi}
\end{array}\right]}_{\mathbf{B}} \mathbf{R}_{\eta} \mathbf{A}^{H}=\mathbf{B R}_{\eta} \mathbf{A}^{H} .
$$

The propagator method relies on the partition of the matrix $\mathbf{B}$ [34]. $\mathbf{B}$ can be denoted by $\mathbf{B}=\left[\begin{array}{l}\mathbf{B}_{1} \\ \mathbf{B}_{2}\end{array}\right]$, where $\mathbf{B}_{1} \in \mathbf{C}^{K \times K}$ 
is the full rank matrix; $\mathbf{B}_{2} \in \mathbf{C}^{(2 M N-K) \times K}$. The propagator $\mathbf{V}$ is a unique linear operator which can be written as $\mathbf{V}^{H} \mathbf{B}_{1}=$ $\mathbf{B}_{2}$. Similarly partitioning received data matrix $\mathbf{R}$ into two submatrices $\mathbf{R}_{1}$ and $\mathbf{R}_{2}$ with dimensions $K \times M N$ and $(2 M N-K) \times M N$, respectively. Then the unique linear operation holds between $\mathbf{R}_{1}$ and $\mathbf{R}_{2}$ :

$$
\mathbf{V}^{H} \mathbf{R}_{1}=\mathbf{R}_{2} .
$$

An estimation matrix $\mathbf{V}$ can be obtained by minimizing the cost function: $J(\mathbf{V})=\min \left\|\mathbf{R}_{2}-\mathbf{V}^{H} \mathbf{R}_{1}\right\|_{F}^{2}$. The optimal solution is given by $\mathbf{V}=\left(\mathbf{R}_{1} \mathbf{R}_{1}^{H}\right)^{-1} \mathbf{R}_{1} \mathbf{R}_{2}^{H}$. Define a new matrix $\widetilde{\mathbf{V}}^{H}=\left[\begin{array}{c}\mathbf{I}_{K} \\ \mathbf{V}^{H}\end{array}\right]$, where $\mathbf{I}_{K}$ is the identity matrix. Combining $\widetilde{\mathbf{V}}$ and (7), we obtain

$$
\widetilde{\mathbf{V}}^{H} \mathbf{R}_{1}=\left[\begin{array}{l}
\mathbf{R}_{1} \\
\mathbf{R}_{2}
\end{array}\right]=\left[\begin{array}{l}
\mathbf{B}_{1} \\
\mathbf{B}_{2}
\end{array}\right] \mathbf{R}_{\eta} \mathbf{A}^{H}=\left[\begin{array}{c}
\mathbf{A} \boldsymbol{\Phi}^{-1} \\
\mathbf{A} \boldsymbol{\Phi}
\end{array}\right] \mathbf{R}_{\eta} \mathbf{A}^{H}=\mathbf{B} \mathbf{R}_{\eta} \mathbf{A}^{H} .
$$

Rewrite (8) as

$$
\widetilde{\mathbf{V}}^{H}=\underbrace{\mathbf{B} \mathbf{R}_{\eta} \mathbf{A}^{H} \mathbf{R}_{1}^{\dagger}}_{\mathbf{T}}=\mathbf{B} \mathbf{T},
$$

where $\mathbf{R}_{1}^{\dagger}$ is the pseudoinverse of $\mathbf{R}_{1}$ and $\mathbf{T}$ is a nonsingular matrix. From (9), the columns in $\widetilde{\mathbf{V}}$ span the same signal subspace as the column vectors in $\mathbf{B}$. So the signal subspace can be obtained by avoiding the estimation and eigen decomposition of the sample covariance matrix. The matrix $\widetilde{\mathbf{V}}^{H}$ can be partitioned into two submatrices $\widetilde{\mathbf{V}}^{H}=\left[\begin{array}{l}\widetilde{\mathbf{V}}_{1} \\ \widetilde{\mathbf{V}}_{2}\end{array}\right]$. According to (9), we can get

$$
\left[\begin{array}{l}
\widetilde{\mathbf{V}}_{1} \\
\widetilde{\mathbf{V}}_{2}
\end{array}\right]=\underbrace{\left[\begin{array}{c}
\mathbf{A} \boldsymbol{\Phi}^{-1} \\
\mathbf{A} \boldsymbol{\Phi}
\end{array}\right]}_{\mathbf{B}} \mathbf{T} .
$$

Notice that $\boldsymbol{\Phi}=\boldsymbol{\Phi}^{-1} \boldsymbol{\Phi}^{2}$; from (10) we can obtain

$$
\begin{gathered}
\boldsymbol{\Phi}^{2}=\mathbf{T} \widetilde{\mathbf{V}}_{1}^{\dagger} \widetilde{\mathbf{V}}_{2} \mathbf{T}^{-1}, \\
\mathbf{A} \boldsymbol{\Phi}=\widetilde{\mathbf{V}}_{2} \mathbf{T}^{-1} .
\end{gathered}
$$

Equation (11) shows that the main diagonal elements of $\boldsymbol{\Phi}^{2}$ are equal to the eigen values obtained via the eigen decomposition of $\widetilde{\mathbf{V}}_{1}^{\dagger} \widetilde{\mathbf{V}}_{2}$ with $\mathbf{T}$ the corresponding eigenvectors. Thus the Doppler frequency of the $k$ th target can be calculated as

$$
\widehat{f}_{k}=\frac{\operatorname{angle}\left(\varphi_{k}\right) f_{s}}{4 \pi}
$$

where $\varphi_{k}$ is the $k$ th diagonal element of $\Phi^{2}$. The $\widehat{\mathbf{A}}$ can be calculated from (12), because the $\Phi$ is the diagonal matrix, and it cannot affect the estimation of DOD and DOA using the least square method from the matrix $\widehat{\mathbf{A}}$. We note that the pairing is automatically obtained because the DOA-DODs and Doppler frequencies are given through the corresponding eigenvectors. The matrix $\widehat{\mathbf{A}}$ can be also denoted by

$$
\widehat{\mathbf{A}}=\widehat{\mathbf{A}}_{R} \circ \widehat{\mathbf{A}}_{T}=\left[\begin{array}{c}
\widehat{\mathbf{A}}_{T} D_{1}\left(\widehat{\mathbf{A}}_{R}\right) \\
\widehat{\mathbf{A}}_{T} D_{2}\left(\widehat{\mathbf{A}}_{R}\right) \\
\vdots \\
\widehat{\mathbf{A}}_{T} D_{N}\left(\widehat{\mathbf{A}}_{R}\right)
\end{array}\right]=\left[\begin{array}{c}
\widehat{\mathbf{A}}_{T} \\
\widehat{\mathbf{A}}_{T} \widehat{\boldsymbol{\Phi}}_{r} \\
\vdots \\
\widehat{\mathbf{A}}_{T} \widehat{\boldsymbol{\Phi}}_{r}^{N-1}
\end{array}\right],
$$

where $\widehat{\mathbf{A}}_{T}=\left[\widehat{\mathbf{a}}_{t}\left(\phi_{1}\right), \widehat{\mathbf{a}}_{t}\left(\phi_{2}\right), \ldots, \widehat{\mathbf{a}}_{t}\left(\phi_{K}\right)\right]$ and $\widehat{\mathbf{A}}_{R}=\left[\widehat{\mathbf{a}}_{r}\left(\theta_{1}\right)\right.$, $\left.\widehat{\mathbf{a}}_{r}\left(\theta_{2}\right), \ldots, \widehat{\mathbf{a}}_{r}\left(\theta_{K}\right)\right]$ are the transmit and receive direction matrices, respectively:

$$
\begin{gathered}
\widehat{\boldsymbol{\Phi}}_{r}=\operatorname{diag}\left[\exp \left(-j \pi \sin \widehat{\theta}_{1}\right), \exp \left(-j \pi \sin \widehat{\theta}_{2}\right), \ldots,\right. \\
\left.\exp \left(-j \pi \sin \widehat{\theta}_{K}\right)\right] .
\end{gathered}
$$

There exists an $M N \times M N$ transformation matrix $\mathbf{C}$ corresponding to the finite number of row interchanged operations such that

$$
\widehat{\mathbf{F}}=\mathbf{C} \widehat{\mathbf{A}}=\widehat{\mathbf{A}}_{T} \circ \widehat{\mathbf{A}}_{R}=\left[\begin{array}{c}
\widehat{\mathbf{A}}_{R} D_{1}\left(\widehat{\mathbf{A}}_{T}\right) \\
\widehat{\mathbf{A}}_{R} D_{2}\left(\widehat{\mathbf{A}}_{T}\right) \\
\vdots \\
\widehat{\mathbf{A}}_{R} D_{M}\left(\widehat{\mathbf{A}}_{T}\right)
\end{array}\right]=\left[\begin{array}{c}
\widehat{\mathbf{A}}_{R} \\
\widehat{\mathbf{A}}_{R} \widehat{\boldsymbol{\Phi}}_{t} \\
\vdots \\
\widehat{\mathbf{A}}_{R} \widehat{\boldsymbol{\Phi}}_{t}^{M-1}
\end{array}\right],
$$

where

$$
\begin{gathered}
\widehat{\Phi}_{t}=\operatorname{diag}\left[\exp \left(-j \pi \sin \widehat{\phi}_{1}\right), \exp \left(-j \pi \sin \widehat{\phi}_{2}\right), \ldots,\right. \\
\left.\exp \left(-j \pi \sin \widehat{\phi}_{K}\right)\right] .
\end{gathered}
$$

We define

$$
\begin{gathered}
\mathbf{P}_{b}=\left[\left(\widehat{\mathbf{A}}_{T} \boldsymbol{\Phi}_{r}\right)^{T},\left(\widehat{\mathbf{A}}_{T} \boldsymbol{\Phi}_{r}^{2}\right)^{T}, \ldots,\left(\widehat{\mathbf{A}}_{T} \boldsymbol{\Phi}_{r}^{N-1}\right)^{T}\right]^{T}, \\
\mathbf{P}_{a}=\left[\left(\widehat{\mathbf{A}}_{T}\right)^{T},\left(\widehat{\mathbf{A}}_{T} \boldsymbol{\Phi}_{r}\right)^{T}, \ldots,\left(\widehat{\mathbf{A}}_{T} \boldsymbol{\Phi}_{r}^{N-2}\right)^{T}\right]^{T} .
\end{gathered}
$$

Owing to $\mathbf{P}_{a} \widehat{\boldsymbol{\Phi}}_{r}=\mathbf{P}_{b}, \widehat{\boldsymbol{\Phi}}_{r}=\mathbf{P}_{a}^{\dagger} \mathbf{P}_{b}$, then we get the estimation of DOA:

$$
\widehat{\theta}_{k}=\sin ^{-1}\left(\frac{\operatorname{angle}\left(p_{k}\right)}{\pi}\right),
$$

where $p_{k}$ is the $k$ th diagonal element of the matrix $\widehat{\Phi}_{r}$.

We also define

$$
\begin{gathered}
\mathbf{B}_{b}=\left[\left(\widehat{\mathbf{A}}_{R} \boldsymbol{\Phi}_{t}\right)^{T},\left(\widehat{\mathbf{A}}_{R} \boldsymbol{\Phi}_{t}^{2}\right)^{T}, \ldots,\left(\widehat{\mathbf{A}}_{R} \boldsymbol{\Phi}_{t}^{N-1}\right)^{T}\right]^{T}, \\
\mathbf{B}_{a}=\left[\left(\widehat{\mathbf{A}}_{R}\right)^{T},\left(\widehat{\mathbf{A}}_{R} \boldsymbol{\Phi}_{t}\right)^{T}, \ldots,\left(\widehat{\mathbf{A}}_{R} \boldsymbol{\Phi}_{t}^{N-2}\right)^{T}\right]^{T},
\end{gathered}
$$

Owing to $\mathbf{B}_{a} \widehat{\boldsymbol{\Phi}}_{t}=\mathbf{B}_{b}, \widehat{\boldsymbol{\Phi}}_{t}=\mathbf{B}_{a}{ }^{\dagger} \mathbf{B}_{b}$, we can also get the estimation of DOD:

$$
\widehat{\phi}_{k}=\sin ^{-1}\left(\frac{\operatorname{angle}\left(\lambda_{k}\right)}{\pi}\right) .
$$

where $\lambda_{k}$ is the $k$ th diagonal element of the matrix $\widehat{\Phi}_{t}$.

Now we show the major steps of the proposed algorithm as follows. 
(1) Compute the covariance matrix of the received data through (5).

(2) Estimate the propagator $\mathbf{V}$ from (7).

(3) Compute the Doppler frequency according to (10), (11), and (13).

(4) Estimate matrix $\widehat{\mathbf{A}}$ from (12); then use the least square method to estimate the DOD and DOA according to (14) (21).

Remark 1. In fact completely orthogonal signals cannot be found in reality, if we consider the transmitted nonorthogonal signals; that is, $\overline{\mathbf{a}}_{t}\left(\phi_{k}\right) \neq \mathbf{a}_{t}\left(\phi_{k}\right)$, and the output signal can be expressed as $\mathbf{x}(t)=\widetilde{\mathbf{A}} \boldsymbol{\eta}(t)+\mathbf{n}(t)$, where $\widetilde{\mathbf{A}}=$ $\left[\widetilde{\mathbf{a}}_{1}, \widetilde{\mathbf{a}}_{2}, \ldots, \widetilde{\mathbf{a}}_{K}\right]$ and $\widetilde{\mathbf{a}}_{k}=\mathbf{a}_{r}\left(\theta_{k}\right) \otimes \overline{\mathbf{a}}_{t}\left(\phi_{k}\right)$. Define $\widetilde{\mathbf{A}}_{T}=$ $\left[\overline{\mathbf{a}}_{t}\left(\phi_{1}\right), \overline{\mathbf{a}}_{t}\left(\phi_{2}\right), \ldots, \overline{\mathbf{a}}_{t}\left(\phi_{K}\right)\right], \widetilde{\mathbf{A}}_{T}=\mathbf{R}_{s} \mathbf{A}_{T}, \widetilde{\mathbf{A}}=\mathbf{A}_{R} \circ$ $\widetilde{\mathbf{A}}_{T}=\overline{\mathbf{R}}_{s}\left[\mathbf{A}_{T}^{T},\left(\mathbf{A}_{T} \boldsymbol{\Phi}_{r}\right)^{T}, \ldots,\left(\mathbf{A}_{T} \boldsymbol{\Phi}_{r}^{N-1}\right)^{T}\right]^{T}$, where $\overline{\mathbf{R}}_{s}=$ $\operatorname{diag}\left[\mathbf{R}_{s}, \mathbf{R}_{s}, \ldots, \mathbf{R}_{s}\right]$. The estimation of $\widetilde{\mathbf{A}}$ can be denoted as $\widehat{\mathbf{A}}=\widetilde{\mathbf{A}} \boldsymbol{\Pi}=\overline{\mathbf{R}}_{s} \mathbf{A} \Pi, \Pi^{-1}=\boldsymbol{\Pi}$. Estimating the matrix $\widetilde{\mathbf{A}}$ from (12), then multiplying $\left(\overline{\mathbf{R}}_{s}\right)^{-1}$, we get $\overline{\mathbf{A}}=\mathbf{A} \Pi$, using the least square method to estimate the DOD and DOA according to (14) (21).

3.2. Complexity Analysis. In contrast to ESPRIT algorithm [31], our algorithm has a low computational load; the main computational cost of our algorithm is the estimation of the matrix $\widetilde{\mathbf{V}}^{H}$, which takes $O\left(2 M^{2} N^{2} K+M N K^{2}+K^{3}\right)$; the total computational complexity of our algorithm is $O\left[M^{2} N^{2}(L-\right.$ 1) $\left.+2 M^{2} N^{2} K+2 M N K^{2}+2 K^{3}+M(N-1) K^{2}+N(M-1) K^{2}\right]$, while ESPRIT requires $O\left(4(L-1) M^{2} N^{2}+8 M^{3} N^{3}+2 K^{3}+\right.$ $\left.M(N-1) K^{2}+N(M-1) K^{2}\right)$ in the eigen decomposition of the covariance matrices. DOA matrix algorithm [32] requires $O\left(2(L-1) M^{2} N^{2}+2 M^{3} N^{3}+M(N-1) K^{2}+N(M-1) K^{2}\right)$. Figure 1 shows the complexity comparison with $M=9$, $N=9, K=3$, and different $L$. From Figure 1 we find that our algorithm has much lower computational load than ESPRIT algorithm and DOA matrix algorithm. The computational cost (CPU time) of the proposed algorithm is $0.053029 \mathrm{~s}$, while ESPRIT algorithm and DOA matrix algorithm need $0.210587 \mathrm{~s}$ and $0.121686 \mathrm{~s}$, respectively, at $M=9, N=9$, $K=3$, and $L=100$ with CPU frequency $2.20 \mathrm{GHz}$.

\subsection{Discussion}

(1) From Figure 1 we find that our algorithm has much lower computational load than ESPRIT algorithm and DOA matrix algorithm. ESPRIT algorithm employs either eigen-value decomposition (EVD) of cross-correlation matrix or singular value. Using the techniques, the computational complexity is very high. Reference [34] has shown the propagator method (PM) for array signal processing to estimate DOA of incident signals without eigen-value decomposition of cross-correlation matrix of the received data. In our proposed algorithm, propagator $\mathbf{V}$ is a linear operator which can easily be extracted from the data matrix R. But the construction of matrix $\widetilde{\mathbf{V}}$ leads

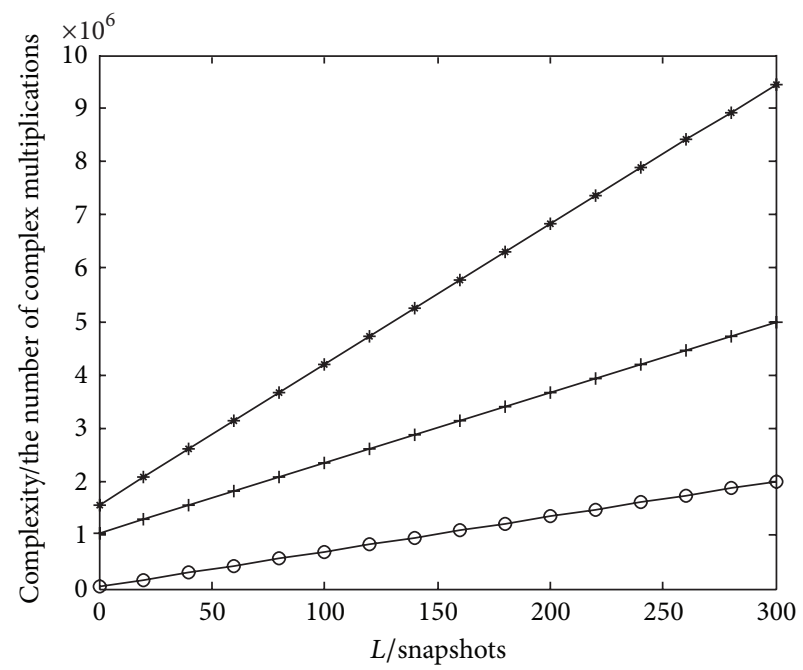

$\rightarrow$ ESPRIT algorithm [31] $\quad$ - The proposed algorithm

, DOA matrix algorithm [32]

Figure 1: Complexity comparison with $M=9, N=9, K=3$, and different $L$.

the proposed algorithm's performance to degrade in low SNR. So the proposed algorithm has very close parameters estimation to ESPRIT algorithm and DOA matrix algorithm in high SNR.

(2) Since the DOA-DODs and Doppler frequencies are given through the corresponding eigenvectors, it can achieve automatically paired estimation of angles and Doppler frequencies.

(3) The proposed algorithm can eliminate the effect of the spatial colored noise since the new matrix is constructed by (5) and (6).

3.4. The Cramér-Rao Bound (CRB). In this section, we derive $\mathrm{CRB}$ of parameter estimation for MIMO radar and rewrite the received data as

$$
\mathbf{Z}=\operatorname{row}(\mathbf{X})=\mathbf{K}\left(f_{d}, \phi, \theta\right) \boldsymbol{\beta}+\mathbf{W}
$$

where $\mathbf{K}\left(f_{d}, \phi, \theta\right)=\left[\mathbf{K}_{1}, \mathbf{K}_{2}, \ldots, \mathbf{K}_{K}\right], \mathbf{K}_{k}=\mathbf{a}_{f}\left(f_{d k}\right) \otimes$ $\mathbf{a}_{k}, \mathbf{a}_{f}\left(f_{d k}\right)=\left[1, e^{j 2 \pi f_{d k} / f_{s}}, \ldots, e^{j 2 \pi f_{d k}(L-1) / f_{s}}\right]^{T}, \boldsymbol{\beta}=\left[\sqrt{P} \beta_{1}\right.$, $\left.\ldots, \sqrt{P} \beta_{K}\right]^{T}$, and $\mathbf{W}$ represents the noise vector. The fisher information matrix (FIM) with respect to $\phi=\left[\phi_{1}, \phi_{2}, \ldots, \phi_{K}\right], \theta=\left[\theta_{1}, \theta_{2}, \ldots, \theta_{K}\right]$, and $f_{d}=\left[f_{d 1}, f_{d 2}\right.$ $\left., \ldots, f_{d K}\right]$ can be calculated as follows $[31,35]$ :

$$
\mathbf{F}=\left[\begin{array}{lll}
\mathbf{F}_{11} & \mathbf{F}_{12} & \mathbf{F}_{13} \\
\mathbf{F}_{21} & \mathbf{F}_{22} & \mathbf{F}_{23} \\
\mathbf{F}_{31} & \mathbf{F}_{32} & \mathbf{F}_{33}
\end{array}\right],
$$




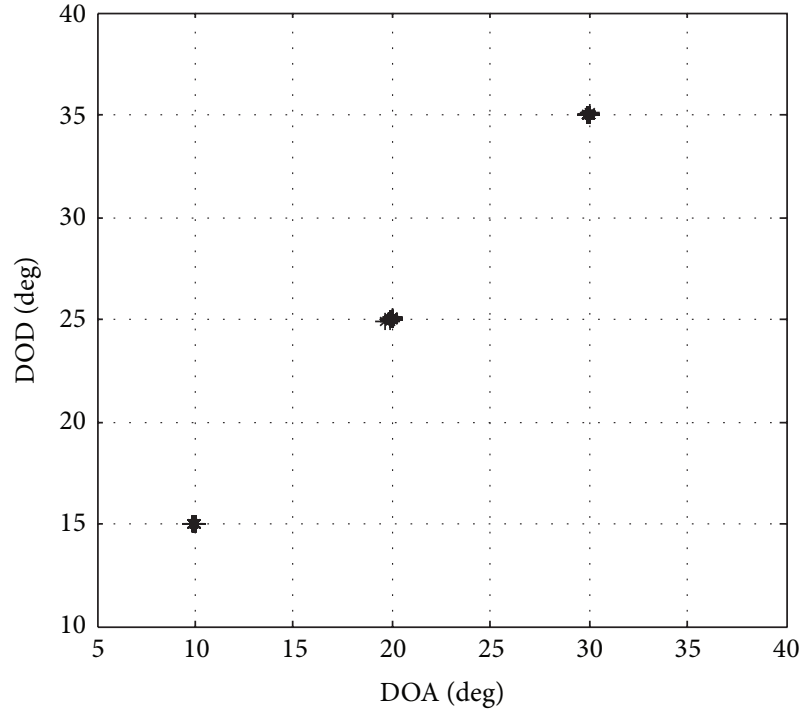

(a)

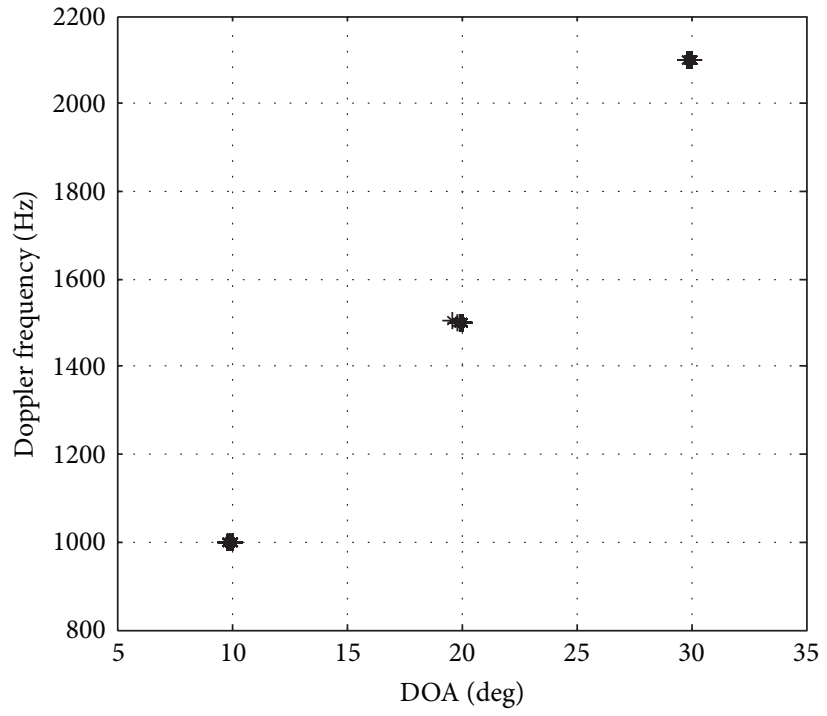

(b)

Figure 2: Angle and Doppler frequency estimation at $\mathrm{SNR}=10 \mathrm{~dB}$.

where

$$
\begin{aligned}
& \mathbf{F}_{11}=2 \operatorname{Re}\left[\boldsymbol{\Gamma}^{H}\left(\mathbf{K}_{\theta}^{\prime}\right)^{H} \boldsymbol{\Pi}^{H} \mathbf{Q}^{-1} \boldsymbol{\Pi} \mathbf{K}_{\theta}^{\prime} \boldsymbol{\Gamma}\right], \\
& \mathbf{F}_{12}=2 \operatorname{Re}\left[\boldsymbol{\Gamma}^{H}\left(\mathbf{K}_{\theta}^{\prime}\right)^{H} \boldsymbol{\Pi}^{H} \mathbf{Q}^{-1} \boldsymbol{\Pi} \mathbf{K}_{\phi}^{\prime} \boldsymbol{\Gamma}\right], \\
& \mathbf{F}_{13}=2 \operatorname{Re}\left[\boldsymbol{\Gamma}^{H}\left(\mathbf{K}_{\theta}^{\prime}\right)^{H} \boldsymbol{\Pi}^{H} \mathbf{Q}^{-1} \boldsymbol{\Pi} \mathbf{K}_{f}^{\prime} \boldsymbol{\Gamma}\right], \\
& \mathbf{F}_{21}=2 \operatorname{Re}\left[\boldsymbol{\Gamma}^{H}\left(\mathbf{K}_{\phi}^{\prime}\right)^{H} \boldsymbol{\Pi}^{H} \mathbf{Q}^{-1} \boldsymbol{\Pi} \mathbf{K}_{\theta}^{\prime} \boldsymbol{\Gamma}\right], \\
& \mathbf{F}_{22}=2 \operatorname{Re}\left[\boldsymbol{\Gamma}^{H}\left(\mathbf{K}_{\phi}^{\prime}\right)^{H} \boldsymbol{\Pi}^{H} \mathbf{Q}^{-1} \boldsymbol{\Pi} \mathbf{K}_{\phi}^{\prime} \boldsymbol{\Gamma}\right], \\
& \mathbf{F}_{23}=2 \operatorname{Re}\left[\boldsymbol{\Gamma}^{H}\left(\mathbf{K}_{\phi}^{\prime}\right)^{H} \boldsymbol{\Pi}^{H} \mathbf{Q}^{-1} \boldsymbol{\Pi} \mathbf{K}_{f}^{\prime} \boldsymbol{\Gamma}\right], \\
& \mathbf{F}_{31}=2 \operatorname{Re}\left[\boldsymbol{\Gamma}^{H}\left(\mathbf{K}_{f}^{\prime}\right)^{H} \boldsymbol{\Pi}^{H} \mathbf{Q}^{-1} \boldsymbol{\Pi} \mathbf{K}_{\theta}^{\prime} \boldsymbol{\Gamma}\right], \\
& \mathbf{F}_{32}=2 \operatorname{Re}\left[\boldsymbol{\Gamma}^{H}\left(\mathbf{K}_{f}^{\prime}\right)^{H} \boldsymbol{\Pi}^{H} \mathbf{Q}^{-1} \boldsymbol{\Pi} \mathbf{K}_{\phi}^{\prime} \boldsymbol{\Gamma}\right], \\
& \mathbf{F}_{33}=2 \operatorname{Re}\left[\boldsymbol{\Gamma}^{H}\left(\mathbf{K}_{f}^{\prime}\right)^{H} \boldsymbol{\Pi}^{H} \mathbf{Q}^{-1} \boldsymbol{\Pi} \mathbf{K}_{f}^{\prime} \boldsymbol{\Gamma}\right],
\end{aligned}
$$

and $\boldsymbol{\Gamma}=\operatorname{diag}(\boldsymbol{\beta})$ denotes the diagonal matrix constructed by the vector $\beta$ :

$$
\begin{gathered}
\mathbf{Q}=\mathbf{I}_{M L} \otimes \mathbf{Q}_{w}, \quad \boldsymbol{\Pi}=\mathbf{I}_{M N}-\mathbf{K}\left(\mathbf{K}^{H} \mathbf{Q}^{-1} \mathbf{K}\right)^{-1} \mathbf{K}^{H} \mathbf{Q}^{-1}, \\
\mathbf{K}_{\theta}^{\prime} \\
=\left[\mathbf{a}_{f}\left(f_{d 1}\right) \otimes\left[\mathbf{a}_{t}\left(\phi_{1}\right) \otimes \frac{\partial \mathbf{a}_{r}\left(\theta_{1}\right)}{\partial \theta_{1}}\right], \ldots,\right. \\
\left.\mathbf{a}_{f}\left(f_{d K}\right) \otimes\left[\mathbf{a}_{t}\left(\phi_{K}\right) \otimes \frac{\partial \mathbf{a}_{r}\left(\theta_{K}\right)}{\partial \theta_{K}}\right]\right],
\end{gathered}
$$

$$
\begin{aligned}
& \mathbf{K}_{\phi}^{\prime} \\
& =\left[\mathbf{a}_{f}\left(f_{d 1}\right) \otimes\left[\frac{\partial \mathbf{a}_{t}\left(\phi_{1}\right)}{\partial \phi_{1}} \otimes \mathbf{a}_{r}\left(\theta_{1}\right)\right], \ldots,\right. \\
& \left.\quad \mathbf{a}_{f}\left(f_{d K}\right) \otimes\left[\frac{\partial \mathbf{a}_{t}\left(\phi_{K}\right)}{\partial \phi_{K}} \otimes \mathbf{a}_{r}\left(\theta_{K}\right)\right]\right] \\
& \mathbf{K}_{f}^{\prime} \\
& =\frac{\partial \mathbf{a}_{f}\left(f_{d 1}\right)}{\partial f_{d 1}} \otimes\left[\mathbf{a}_{t}\left(\phi_{1}\right) \otimes \mathbf{a}_{r}\left(\theta_{1}\right)\right], \ldots, \\
& \left.\quad \frac{\partial \mathbf{a}_{f}\left(f_{d K}\right)}{\partial f_{d K}} \otimes\left[\mathbf{a}_{t}\left(\phi_{K}\right) \otimes \mathbf{a}_{r}\left(\theta_{K}\right)\right]\right]
\end{aligned}
$$

Then the CRB matrix is

$$
\mathrm{CRB}=\mathbf{F}^{-1} \text {. }
$$

\section{Simulation Results}

We present the Monte Carlo simulations to assess the parameter estimation performance of our algorithm. Define root mean squared error (RMSE) as (1/ K) $\sum_{k=1}^{K} \sqrt{(1 / 1000) \sum_{n=1}^{1000}\left[\left(\widehat{\phi}_{k, n}-\phi_{k}\right)^{2}+\left(\hat{\theta}_{k, n}-\theta_{k}\right)^{2}\right]}$, where $\widehat{\theta}_{k, n}$ is the estimate of DOA $\theta_{k}$ of the $n$th Monte Carlo trial and $\widehat{\phi}_{k, n}$ is the estimate of DOD $\phi_{k}$ of the $n$th Monte Carlo trial. Define RMSE of Doppler frequency as $(1 / K) \sum_{k=1}^{K} \sqrt{(1 / 1000) \sum_{n=1}^{1000}\left(\widehat{f}_{k, n}-f_{k}\right)^{2}}$, where $\widehat{f}_{k, n}$ is the estimate of Doppler frequency $f_{k}$ of the $n$th Monte Carlo trial. Note that $M, N, L$, and $K$ are the number of transmit antennas, the receive antennas, the snapshots of the targets, and the number of the targets, respectively. We assume that 


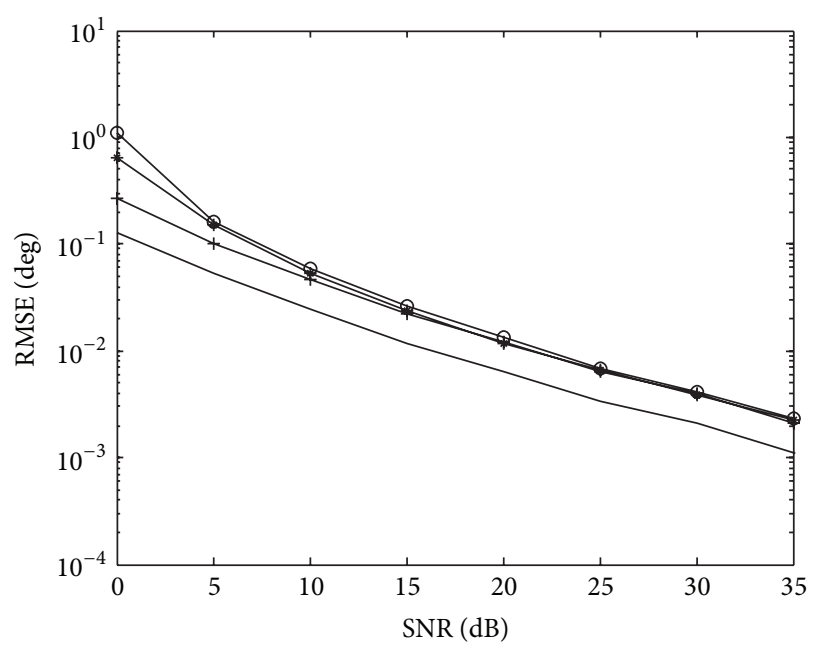

* ESPRIT algorithm [31] $\quad-\quad$ The proposed algorithm

+ DOA matrix algorithm [32] — CRB

FIGURE 3: Angle estimation comparison performance.

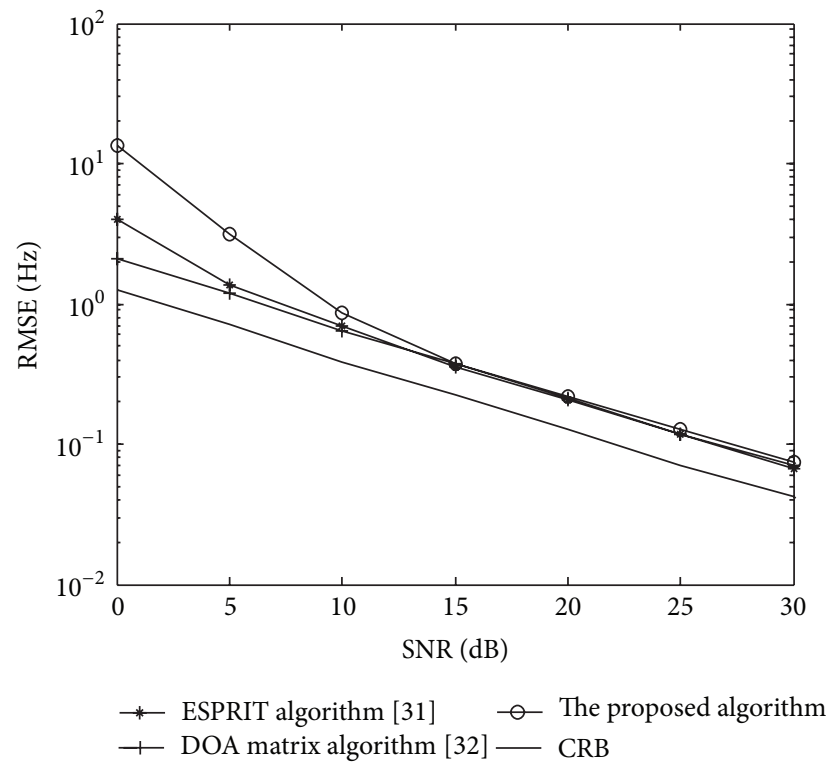

FIGURE 4: Doppler frequency estimation comparison.

there are $K=3$ independent targets, which are located at angles $\left(\theta_{1}, \phi_{1}\right)=\left(10^{\circ}, 15^{\circ}\right),\left(\theta_{2}, \phi_{2}\right)=\left(20^{\circ}, 25^{\circ}\right)$, and $\left(\theta_{3}, \phi_{3}\right)=\left(30^{\circ}, 35^{\circ}\right)$, respectively. The Doppler frequencies of the three targets are $1000 \mathrm{~Hz}, 1500 \mathrm{~Hz}$, and $2100 \mathrm{~Hz}$, respectively, and their RCS are given by $\beta_{1}=\beta_{2}=\beta_{3}=1$. The pulse repeat frequency $f_{s}$ is $10 \mathrm{KHz}$ for a $M=9$ and $N=9$ bistatic MIMO radar. The $(m, n)$ th element of the unknown noise covariance matrix $\mathbf{Q}_{w}$ is $0.9^{|m-n|} e^{j \pi(m-n) / 2}$. Figure 2 shows the estimation results with 100 Monte Carlo trials at $L=100$. As seen in Figure 2, the DODs, DOAs, and Doppler frequencies of all three targets are correctly paired and well localised.

Figures 3 and 4 show the angle and Doppler frequency estimation performance comparison with $M=9, N=9$,

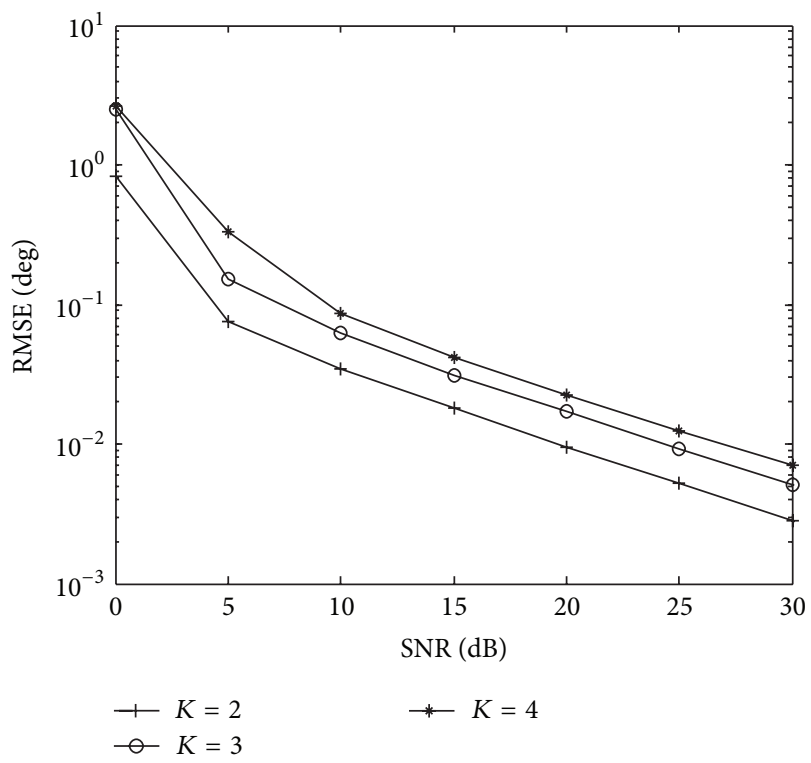

FIgUre 5: Angle estimation with different values of $K$.

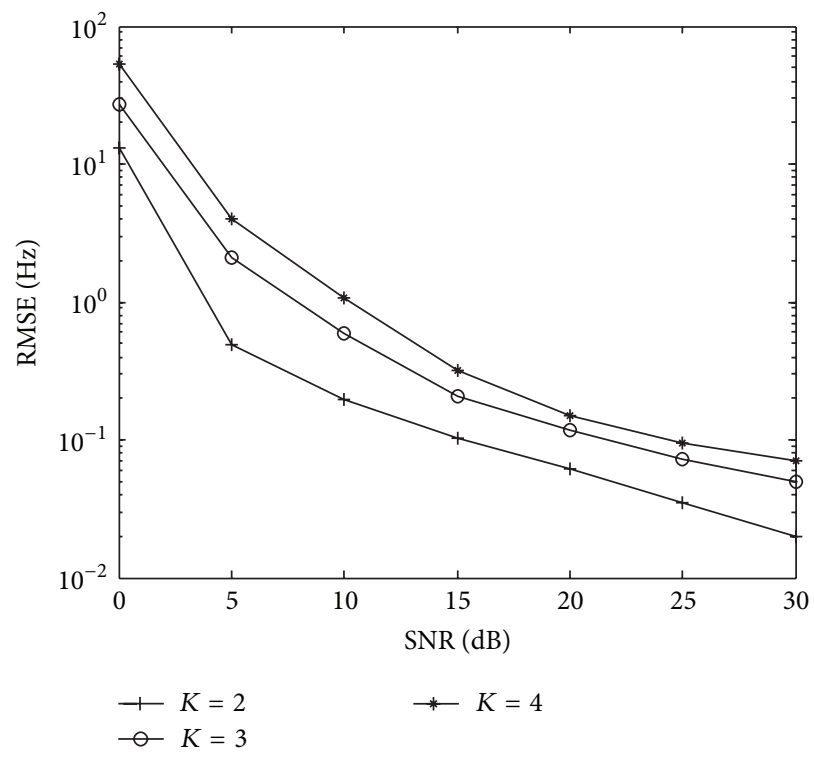

FIgURE 6: Doppler frequency estimation with different values of $K$.

$K=3$, and $L=100$, where we compare our algorithm with ESPRIT algorithm [31], DOA matrix algorithm [32], and CRB. It is indicated in Figures 3 and 4 that our algorithm has very close parameter estimation performance to ESPRIT algorithm and DOA matrix algorithm in high SNR condition.

The simulation of Figures 5 and 6 investigates the performance of our proposed algorithm under different number of targets $K$. The number of targets is set as 2 and 4 . Our proposed algorithm has the different performance under different $K$, as shown in Figures 5 and 6 where $M=9, N=9$, and $L=100$ are considered. From Figures 5 and 6 we find that angle estimation performance of our proposed algorithm degrades with $K$ increasing. 


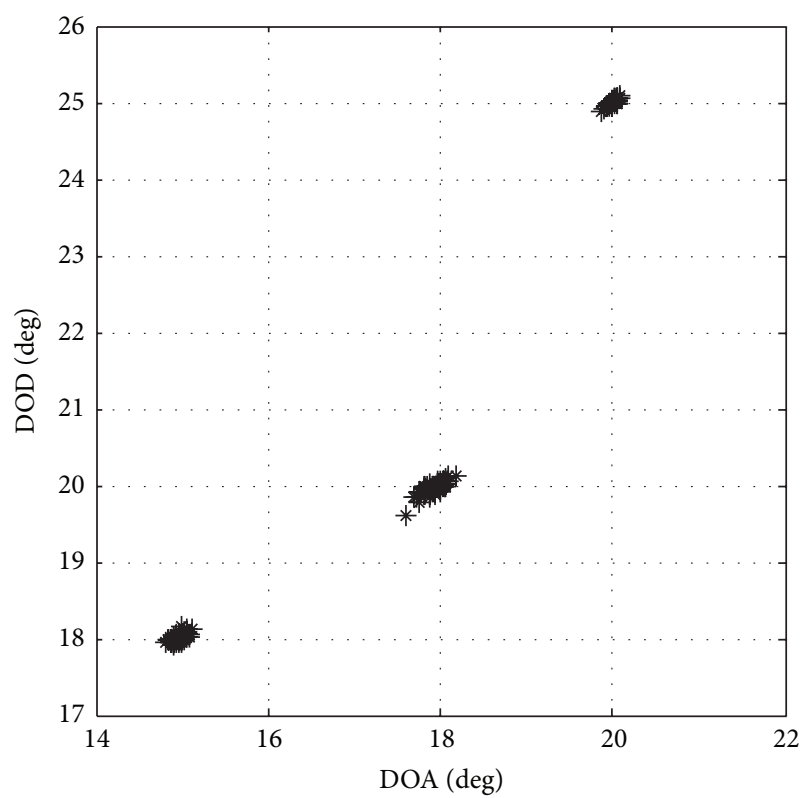

(a)

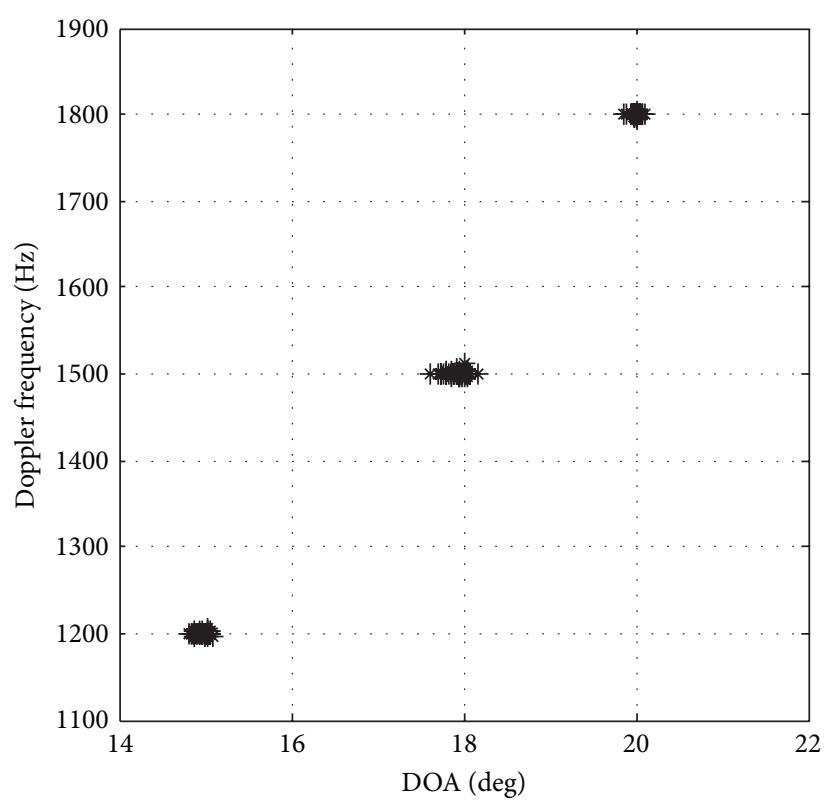

(b)

FIGURE 7: Angle and Doppler frequency estimation at $\mathrm{SNR}=12 \mathrm{~dB}$.

Figure 7 presents parameter estimation performance with three closely spaced targets, where $M=8, N=8$, and $L=100$ are considered. The closely spaced targets are located at angles $\left(\theta_{1}, \phi_{1}\right)=\left(15^{\circ}, 18^{\circ}\right),\left(\theta_{2}, \phi_{2}\right)=\left(18^{\circ}, 20^{\circ}\right)$, and $\left(\theta_{3}, \phi_{3}\right)=$ $\left(20^{\circ}, 25^{\circ}\right)$. The Doppler frequencies of the three targets are $1200 \mathrm{~Hz}, 1500 \mathrm{~Hz}$, and $1800 \mathrm{~Hz}$, respectively. From Figure 7, we find that our algorithm works well in the case of closely spaced targets.

\section{Conclusion}

We have presented a low-complexity angle and Doppler frequency estimation based on propagator method for MIMO radar in spatial colored noise. The proposed algorithm can obtain automatically paired transmit and receive angle estimations in the MIMO radar and eliminate the influence of the spatial colored noise. Furthermore, it provides lower computational complexity and has close parameters estimation compared to ESPRIT algorithm and DOA matrix algorithm in high SNR. It is applicable even if the transmitted waveforms are not orthogonal.

\section{Conflict of Interests}

The authors declare that there is no conflict of interests regarding the publication of this paper.

\section{Acknowledgments}

This work is supported by Nanjing University of Aeronautics and Astronautics Research Funding (NZ2013208). The authors are grateful to the anonymous referees for their constructive comments and suggestions in improving the quality of this paper.

\section{References}

[1] E. Fishler, A. Haimovich, R. Blum, D. Chizhik, L. Cimini, and R. Valenzuela, "MIMO radar: an idea whose time has come," in Proceedings of the IEEE Radar Conference, pp. 71-78, April 2004.

[2] J. Li and P. Stoica, "MIMO radar-diversity means superiority," in Proceedings of the 14th Adaptive Sensor Array Process Workshop (ASAP '06), Lincoln Lab, Lexington, Mass, USA, December 2006.

[3] E. Fishler, A. Haimovich, R. S. Blum, L. J. Cimini Jr., D. Chizhik, and R. A. Valenzuela, "Spatial diversity in radars-models and detection performance," IEEE Transactions on Signal Processing, vol. 54, no. 3, pp. 823-838, 2006.

[4] D. R. Fuhrmann and G. S. Antonio, "Transmit beamforming for MIMO radar systems using partial signal correlation," in Proceedings of the 38th Asilomar Conference on Signals, Systems and Computers, vol. 1, no. 12, pp. 295-299, November 2004.

[5] J. Li and P. Stoica, "MIMO radar with colocated antennas," IEEE Signal Processing Magazine, vol. 24, no. 5, pp. 106-114, 2007.

[6] A. M. Haimovich, R. S. Blum, and L. J. Cimini, "MIMO radar with widely separated antennas," IEEE Signal Processing Magazine, vol. 25, no. 1, pp. 116-129, 2008.

[7] M. Jin, G. S. Liao, and J. Li, "Joint DOD and DOA estimation for bistatic MIMO radar," Signal Processing, vol. 89, no. 2, pp. 244-251, 2009.

[8] M. Yang and G. Zhang, "Compressive sensing based parameter estimation for monostatic MIMO noise radar," Progress in Electromagnetics Research Letters, vol. 30, pp. 133-143, 2012.

[9] H. Jiang, Y. Zhang, J. Li, and H. Cui, "A parafac-based algorithm for multidimensional parameter estimation in polarimetric bistatic MIMO radar," EURASIP Journal on Advances in Signal Processing, vol. 2013, article 133, 2013. 
[10] M. L. Bencheikh, Y. Wang, and H. Y. He, "Polynomial root finding technique for joint DOA DOD estimation in bistatic MIMO radar," Signal Processing, vol. 90, no. 9, pp. 2723-2730, 2010.

[11] M. Hatam, A. Sheikhi, and M. A. Masnadi-Shirazi, "Target detection in pulse-train mimo radars applying ICA algorithms," Progress in Electromagnetics Research, vol. 122, pp. 413-435, 2012.

[12] J. C. Ding, H. W. Chen, H. Q. Wang, X. Li, and Z. W. Zhuang, "Low-grazing angle target detection and system conguration of MIMO radar," Progress in Electromagnetics Research B, vol. 48, pp. 23-42, 2013.

[13] M. Yang and G. Zhang, "Parameter identiability of monostatic MIMO chaotic radar using compressed sensing," Progress in Electromagnetics Research B, vol. 44, pp. 367-382, 2012.

[14] C. Chen and X. Zhang, "A low-complexity joint 2D-DOD and 2D-DOA estimation algorithm for MIMO radar with arbitrary arrays," International Journal of Electronics, vol. 100, no. 10, pp. 1455-1469, 2013.

[15] H. Lv, D.-Z. Feng, H.-W. Liu, J. He, and C. Xiang, "Tri-iterative least-square method for bearing estimation in MIMO radar," Signal Processing, vol. 89, no. 12, pp. 2686-2691, 2009.

[16] I. Bekkerman and J. Tabrikian, "Target detection and localization using MIMO radars and sonars," IEEE Transactions on Signal Processing, vol. 54, no. 10, pp. 3873-3883, 2006.

[17] P. Stoica, J. Li, and Y. Xie, "On probing signal design for MIMO radar," IEEE Transactions on Signal Processing, vol. 55, no. 8, pp. 4151-4161, 2007.

[18] J. Li and P. Stoica, MIMO Radar Signal Processing, Wiley-IEEE Press, New York, NY, USA, 2008.

[19] Q. He, R. S. Blum, H. Godrich, and A. M. Haimovich, "Target velocity estimation and antenna placement for MIMO radar with widely separated antennas," IEEE Journal on Selected Topics in Signal Processing, vol. 4, no. 1, pp. 79-100, 2010.

[20] H. W. Chen, Y. P. Chen, X. Li, and Z. W. Zhuang, "Extended ambiguity function for bistatic MIMO radar," Journal of Systems Engineering and Electronics, vol. 23, no. 2, pp. 109-114, 2012.

[21] L. Xu, J. Li, and P. Stoica, "Target detection and parameter estimation for MIMO radar systems," IEEE Transactions on Aerospace and Electronic Systems, vol. 44, no. 3, pp. 927-939, 2008.

[22] J. Li, P. Stoica, L. Xu, and W. Roberts, "On parameter identifiability of MIMO radar," IEEE Signal Processing Letters, vol. 14, no. 12, pp. 968-971, 2007.

[23] L. Xu, J. Li, and P. Stoica, "Adaptive techniques for mimo radar," in Proceedings of the 4th IEEE Sensor Array and Multichannel Signal Processing (SAM '06), pp. 258-262, July 2006.

[24] F. C. Robey, S. Coutts, D. Weikle, J. C. McHarg, and K. Cuomo, "MIMO radar theory and experimental results," in Proceedings of the 38th Asilomar Conference on Signals, Systems and Computers, vol. 1, no. 12, pp. 300-304, November 2004.

[25] C. Duofang, C. Baixiao, and Q. Guodong, "Angle estimation using ESPRIT in MIMO radar," Electronics Letters, vol. 44, no. 12, pp. 770-771, 2008.

[26] Z. Zheng, J. Zhang, and J. Y. Zhang, "Joint DOD and DOA estimation of bistatic MIMO radar in the presence of unknown mutual coupling," Signal Processing, vol. 92, no. 12, pp. 30393048, 2012.

[27] R. Xie, Z. Liu, and J.-X. Wu, "Direction finding with automatic pairing for bistatic MIMO radar," Signal Processing, vol. 92, no. 1, pp. 198-203, 2012.
[28] X. Zhang, H. Wu, J. Li, and D. Xu, "Computationally efficient DOD and DOA estimation for bistatic MIMO radar with propagator method," International Journal of Electronics, vol. 99, no. 9, pp. 1207-1221, 2012.

[29] C. Jinli, G. Hong, and S. Weimin, "Angle estimation using ESPRIT without pairing in MIMO radar," Electronics Letters, vol. 44, no. 24, pp. 1422-1423, 2008.

[30] X. Zhang, L. Xu, L. Xu, and D. Xu, "Direction of departure (DOD) and direction of arrival (DOA) estimation in MIMO radar with reduced-dimension mUSIC," IEEE Communications Letters, vol. 14, no. 12, pp. 1161-1163, 2010.

[31] W.-B. Fu, T. Su, Y.-B. Zhao, and X.-H. He, "Joint estimation of angle and Doppler frequency for bistatic MIMO radar in spatial colored noise," Journal of Electronics \& Information Technology, vol. 33, no. 12, pp. 2858-2862, 2011.

[32] C. Yunhe, "Joint estimation of angle and Doppler frequency for bistatic MIMO radar," Electronics Letters, vol. 46, no. 2, pp. 170172, 2010.

[33] M. L. Bencheikh, Y. Wang, and H. He, "Polynomial root finding technique for joint DOA DOD estimation in bistatic MIMO radar," Signal Processing, vol. 90, no. 9, pp. 2723-2730, 2010.

[34] S. Marcos, A. Marsal, and M. Benidir, "The propagator method for source bearing estimation," Signal Processing, vol. 42, no. 2, pp. 121-138, 1995.

[35] P. Stoica and R. L. Moses, Spectral Analysis of Signals, Prentice Hall, Upper Saddle River, NJ, USA, 2005. 

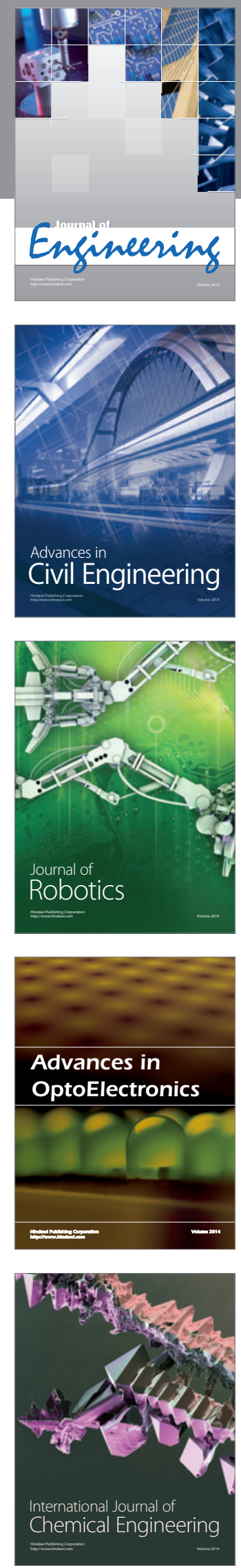

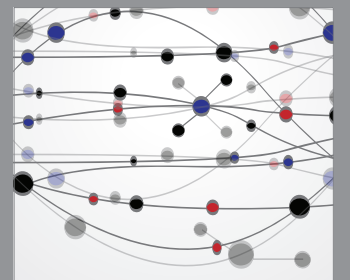

The Scientific World Journal
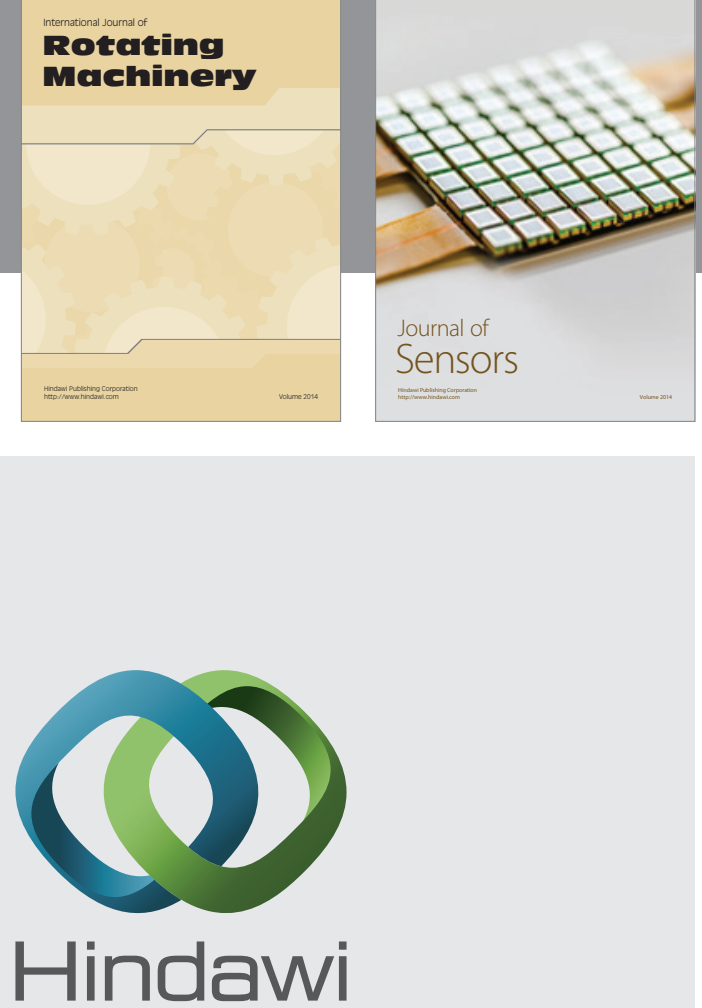

Submit your manuscripts at http://www.hindawi.com
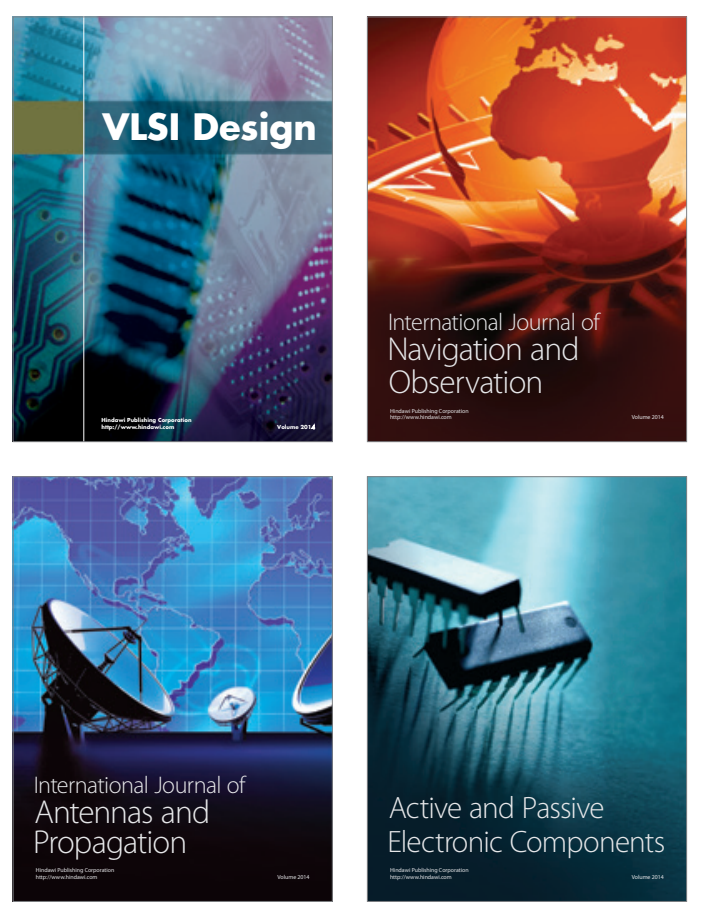
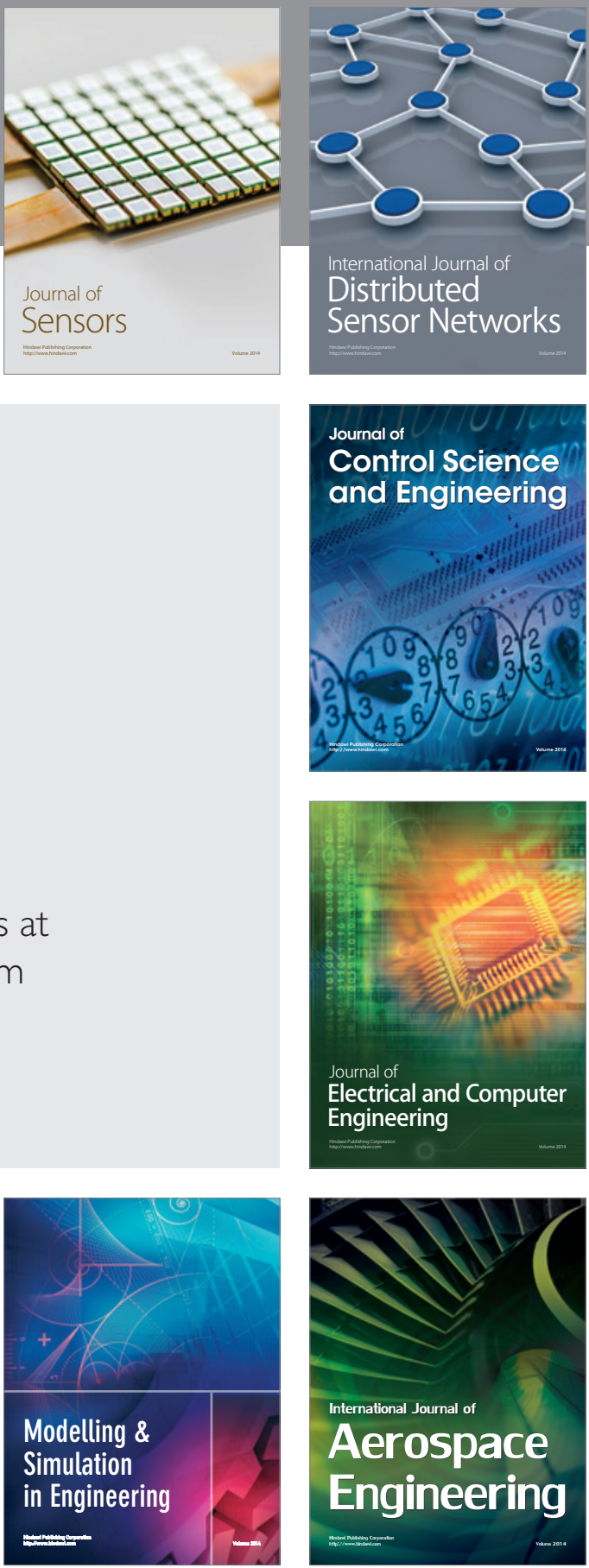

Journal of

Control Science

and Engineering
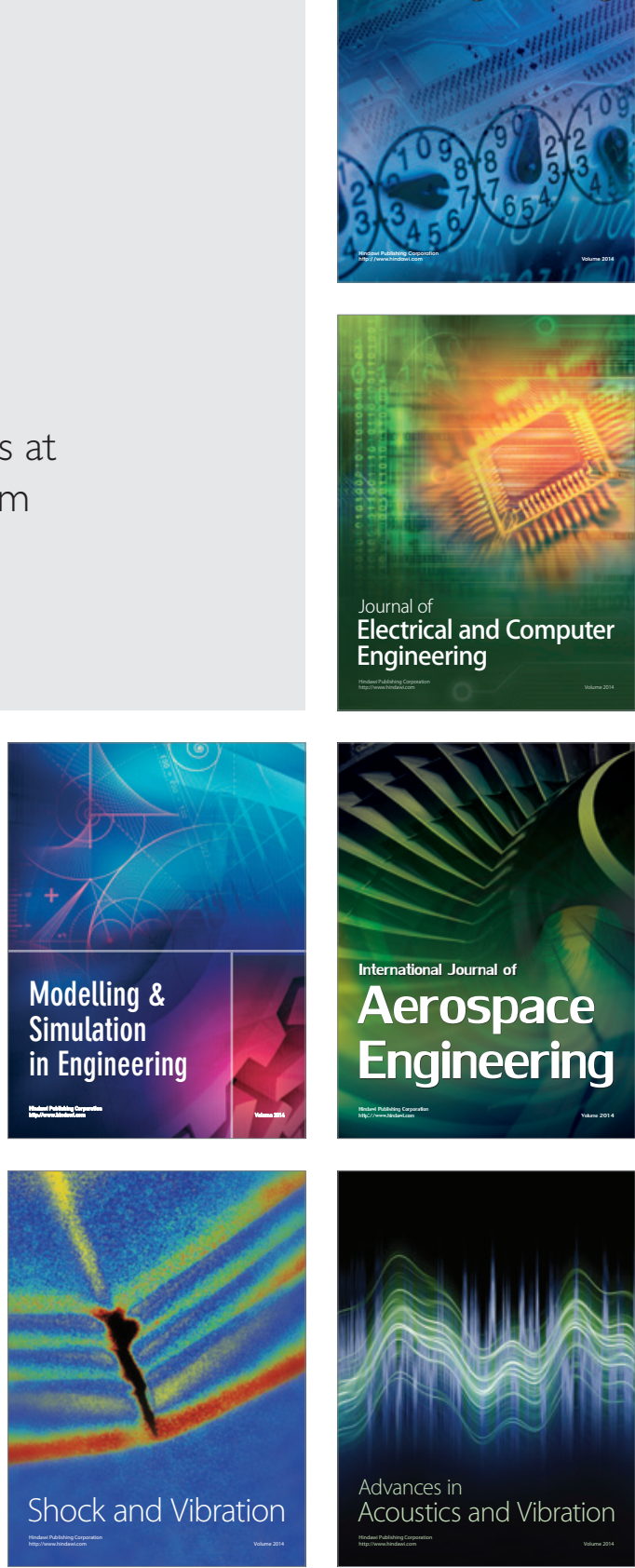